\title{
TRATAMIENTO DE LAS CREENCIAS MOTIVACIONALES EN CONTEXTOS EDUCATIVOS PLURICULTURALES
}

\author{
Francisco Herrera, Mª Inmaculada Ramírez, José Mª Roa e Inmaculada Herrera \\ Universidad de Granada, España
}

\section{INTRODUCCIÓN}

¿Qué hay de cierto en que nuestros alumnos, en general, están desmotivados en sus estudios?. Tan deficiente es la educación que les ofrecemos los "agentes educadores" -profesores, padres, amigos y sociedad- que no somos capaces de atraerlos para sentirse autoconstructores responsables de sus aprendizajes. Pues, si esto es así, deberíamos recordar que una persona que no disfruta con lo que hace, se convierte en un esclavo de sus obligaciones. Acaso, ¿no es nuestra intención formar personas libres, pacíficas y democráticas?, ¿qué está ocurriendo?...

Precisamente, esta investigación quiere ahondar en esa línea, desde la constatación de los niveles de creencias motivacionales de nuestros alumnos y su relación con el rendimiento académico, hasta su posible mejora, a través del diseño y aplicación de un programa de intervención específico.

\section{FINALIDAD DE LA INVESTIGACIÓN}

Teniendo en cuenta las investigaciones más relevantes en este campo, como las de Lepper, Greene y Nisbett (1996) sobre motivación intrínseca, la de Weiner (1986) sobre atribución causal y sus implicaciones en motivación sobre metas de aprendizaje y metas de logro (Alonso, 1991), , las de Eccles (1983) sobre la expectativa-valor de la tarea, mejoradas por Pintrich y De Groot (1990), introduciendo como elementos determinantes los componentes expectativos, valorativos y afectivos -autoeficacia, valor intrínseco y control de la ansiedad-, nuestra intención ha sido la siguiente:

$1^{\circ}$. Analizar el comportamiento de las variables creencias motivacionales y rendimiento académico, en un contexto educativo pluricultural como el de Ceuta, al objeto de comprobar sus niveles y relaciones, particularmente, de cara a la predicción.

$2^{\circ}$. De encontrarse niveles bajos en las creencias motivacionales que pudieran afectar al rendimiento académico, elaborar y aplicar un programa de intervención para mejorar la situación y comprobar su eficacia.

\section{PROBLEMA}

Los principales problemas investigados, como cuestiones prioritarias a tratar, son los siguientes:

Como problema general: ¿Cómo afectan las creencias motivacionales de los alumnos a su rendimiento académico en el contexto educativo pluricultural de Ceuta?

Cuestión a la que, en base a investigaciones precedentes, se podría responder en determinados aspectos; aunque, no es menos cierto que cabría preguntarnos por cuestiones que serían convenientes matizar específicamente con mayor profundidad, tales como por ejemplo: la incidencia del 
factor "cultural". Estas consideraciones nos han llevado a plantear los siguientes problemas específicos:

1․ ¿Qué diferencias estadísticamente significativas existen en las creencias motivacionales "autoeficacia, valor intrínseco y ansiedad» de los alumnos de los cursos $4^{\circ}$ y $6^{\circ}$ de Educación Primaria y $2^{\circ}$ de E.S.O., de los sexos -hombre y mujer- y de las culturas -cristiana y musulmana, en el contexto educativo pluricultural de Ceuta?.

2ํ. ¿Qué diferencias estadísticamente significativas existen en el rendimiento académico de los alum-

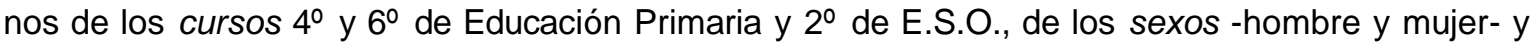
de las culturas -cristiana y musulmana-, en el contexto educativo pluricultural de Ceuta?.

3․ ¿Qué relaciones estadísticamente significativas existen entre las variables analizadas, especialmente, de cara a la predicción?.

$4^{\circ}$. De encontrarse niveles bajos, estadísticamente significativos, en las creencias motivacionales que pudieran afectar al rendimiento académico, ¿es posible elaborar y aplicar un programa de intervención para mejorar la situación y comprobar su eficacia?.

\section{REVISIÓN TEÓRICA}

La motivación constituye una de las grandes claves explicativas de la conducta humana, que, en general, se refiere al porqué del comportamiento (del latín, motus: movimiento; motivación: lo que mueve). Dicho de otra forma, la motivación representa qué es lo que originariamente determina que una persona inicie una acción (activación), se desplace hacia un objetivo (dirección) y persista en sus tentativas para alcanzarlo (mantenimiento).

A grandes rasgos, la evolución histórica de la interpretación acerca de la motivación podríamos resumirla de la siguiente manera:

$1^{\circ}$. Desde los años 20 hasta mediados de los 60 , se caracterizó por el estudio psicoanalítico y del drive, cuyo concepto dominante fue el de homeostasis. La investigación estuvo asociada a la conducta subhumana, particularmente, en la investigación experimental sobre: conducta motora, instinto, impulso, arousal, drive y energetización. Los psicólogos intentaron averiguar qué es lo que mueve a un organismo a restaurar su estado de equilibrio (homeostasis), ocupándose de factores externos como los refuerzos. En la década de los 50, algunos autores, como Lewin, Atkinson y Rutter, impulsaron teorías motivacionales cuasicognitivas, pero siguieron aludiendo a los drives internos y los refuerzos externos como determinantes de la motivación.

2․ A partir de los años 60 , nacen las teorías cognitivas sobre la motivación, centrándose en la experiencia consciente; siendo, a partir de entonces, cuando surge el interés por la motivación de rendimiento, destacando su importancia en la vida de las personas, junto a los logros. Así, la teoría de Atkinson, destacó que la motivación de rendimiento está determinada por el valor dado a la meta y las expectativas de conseguirla, atendiendo a las características de las personas con alta y baja necesidad de rendimiento, ansiedad y control interno.

$3^{\circ}$. Desde los años 70 hasta nuestros días, la pauta viene marcada definitivamente por las teorías cognitivas, decayendo el interés por el estudio de la motivación (Toates, 1986), orientándose a la 
determinación de la importancia de algunos de sus aspectos constitutivos (Vila,1983), destacando el autoconcepto como elemento nuclear de las teorías motivacionales. Los estudios se centran en el papel de la atribución causal, la percepción de competencia, la percepción de control, las estrategias de creencias sobre capacidad y autoeficacia, la indefensión aprendida y un amplio etc. Precisamente, su valor educativo resulta notable, especialmente, porque facilita el conocimiento de la conducta y el rendimiento escolar, así como las líneas maestras necesarias para motivar a los estudiantes, enriquecedoras de la personalidad y eficaces para implicarlos activamente en su propio aprendizaje.

Evidentemente, la motivación no es una variable observable, sino un constructo hipotético (Ball, 1988), una inferencia que hacemos a partir de las manifestaciones de la conducta, pudiendo ser acertada o equivocada, sin que exista una explicación unánime a tal fenómeno, ya que en ello están comprometidos valores sociales que afectan a la clase de persona y a su modelo de sociedad. Así, por ejemplo, cuando el profesor advierte que un estudiante permanece largo tiempo sentado o quieto en su pupitre, puede pensar o inferir que está fuertemente motivado para el aprendizaje, pero también puede pensar que está poco motivado por la falta de actividad aparente. Con todo ello, a pesar de estas interpretaciones subjetivas de la conducta, la motivación supone un acercamiento notable al proceso de explicación científica de la conducta y, sobre todo, constituye un elemento útil y esencial en el ámbito de la acción educativa (Beltrán, 1984: 123).

Una visión excesivamente pragmática y miope de la motivación es la que sitúa al profesor ante la perspectiva de aumentar una determinada conducta haciéndola rápida y persistente. Evidentemente, éste es un objetivo educativo aceptable pero inmediato, a corto plazo, y, de ninguna manera, el único, ni el principal; pues, la acción educativa no debe limitarse a preparar a los sujetos para el examen, o para hacer una demostración brillante ante las autoridades, sino que debe actuar de tal manera que la motivación trascienda del ambiente físico inmediato y se proyecte hacia el futuro, activando los dinamismos del alumno al margen de los incentivos externos habitualmente presentados. Esto quiere decir que la motivación es uno de los factores, junto con la inteligencia y el aprendizaje previo, que determinan si los estudiantes lograrán los resultados académicos apetecidos (Beltrán, 1987:206). En este sentido la motivación es un medio con relación a otros objetivos.

De todas formas, la motivación también constituye, por sí misma, un objetivo de la educación. La sociedad necesita y desea que los estudiantes se sientan interesados por las actividades intelectuales, no sólo durante la etapa de la enseñanza formal, sino también después que ésta haya terminado, lo que se consigue poniendo al estudiante al control de lo que produce; es decir, haciendo que el aprendizaje sea reforzante por sí mismo. El profesor habrá de lograr que los alumnos no sólo mantengan un elevado nivel de regularidad en el estudio de una determinada materia, sino que, incluso, se sientan atraídos hacia esa materia y, para conseguirlo, será preciso que el alumno encuentre reforzante lo que está practicando.

Aunque, por el momento, no existe una definición que reúna las características adecuadas para ser universalmente aceptada, hay, sin embargo, una definición convencional que suele ser generalmente bien admitida, como conjunto de procesos implicados en la activación, dirección y mantenimiento de la conducta. Esta definición tiene el mérito de destacar sus tres dimensiones esenciales: activadora, directiva y persistente; pero también presenta algunas limitaciones, como su carácter de constructo hipotético inferencial, por lo que resulta difícilmente observable. 
En el contexto escolar, el término motivación designa el grado de participación y perseverancia de los alumnos en la tarea, cualquiera que sea la índole de la misma. Por otra parte, la motivación es extremadamente compleja como para reducirla a un proceso unitario, en realidad, representa uno más entre los muchos factores determinantes de la conducta, como son el cognitivo, emocional y actitudinal.

Debido a este carácter complejo y difuso, la motivación mantiene amplias relaciones con otros conceptos también comprometidos con la dirección y la intensidad de la conducta, como son el interés, la necesidad, el valor, la actitud y la aspiración. Mientras que el interés hace referencia a la atención selectiva dentro del campo, la necesidad implica falta o carencia de algo que puede ser suministrado por una determinada actividad.

Más complejo resulta el concepto de valor, que implica una orientación a la meta o metas centrales en la vida de un sujeto. También, la actitud tiene un cierto parentesco con la motivación. En la actitud se distinguen tres componentes: el cognitivo (ideas o creencias acerca de algo o alguien), emocional (sentimientos de agrado o desagrado) y conductual (conducta relacionada con el objeto de la actitud).

Por último, la aspiración se refiere a la expectativa de alcanzar un nivel determinado de logro. El interés, la necesidad, el valor, la actitud y la aspiración dirigen la conducta y controlan el grado o intensidad de la misma en la dirección apuntada.

En el ámbito educativo, es bien conocida la existencia de una correlación positiva entre motivación y rendimiento de una intensidad moderada, aproximadamente de 0,34. De ahí se deduce que una motivación elevada conducirá a un buen rendimiento. Sin embargo, el buen rendimiento también conduce habitualmente a niveles altos de motivación, por lo que resulta arriesgado concluir algo sobre la dirección de la relación causa-efecto; posiblemente la dirección camina en ambos sentidos; es decir, probablemente existe una relación bidireccional.

La motivación ayuda a entender algunos fenómenos escolares curiosos como el infra o supra rendimiento. Como se sabe, la correlación entre inteligencia y rendimiento es de 0,45 , con lo que podemos pensar que, a mayor inteligencia, habrá mayor rendimiento. Ahora bien, al no darse una correlación perfecta, habrá algunos sujetos de baja capacidad con alto rendimiento y, al revés, sujetos de alta capacidad con bajo rendimiento; esto es, precisamente, lo que configura los conceptos de supra-rendimiento y de infrarendimiento; es decir, un rendimiento mayor o menor que el predicho sobre la base de la inteligencia individual. Posiblemente, estos conceptos sólo se explican psicológicamente desde la motivación. El infrarendidor se esfuerza menos y el supra-rendidor se esfuerza más que los estudiantes que obtienen un rendimiento en concordancia con lo predicho respecto a su capacidad intelectual (Beltrán, 1987:207).

Aunque, en realidad, son muchos los factores de la personalidad relacionados con el rendimiento, hoy día se está prestando mucha atención a la motivación académica y sus elementos constituyentes ( $\mathrm{Pa}$ ris, Olson y Stevenson, 1983; Weiner, 1986; McClelland, 1987; Stipek, 1988). No obstante, a pesar de que son muchos los constructos relacionados (locus control, valor dado a las metas, expectativas de éxito, percepción de competencias, atribuciones, ansiedad, etc.), han terminado por integrarse en modelos o teorías sobre la motivación que intentan explicar sus interrelaciones.

Weiner (1986), señala que, entre otros aspectos, toda teoría de la motivación debe contemplar los siguientes: 
- Estar basada sobre otros conceptos más allá del de homeostasis.

- Abarcar bastante más que el hedonismo.

- Incluir todos los procesos cognitivos.

- Tener en cuenta la experiencia consciente.

- Interesarse por el self.

- Integrar un amplio abanico de emociones.

- Construirse sobre el apoyo de relaciones empíricas.

- Explicar las relaciones causales entre cognición, afectividad y comportamiento.

Precisamente, la inclusión del self y el establecimiento de interrelaciones entre la cognición, la afectividad y el comportamiento, son los rasgos más característicos que diferencian las modernas teorías sobre la motivación respecto a las anteriores. Weiner (1990), en este sentido, manifiesta que el hecho más significativo de la moderna psicología ha sido la conceptualización del fenómeno motivacional en términos cognitivos y la inclusión del sí mismo.

En suma, tras una revisión exhaustiva de las definiciones, teorías, sistemas de evaluación y programas de intervención más relevantes sobre la motivación, podemos destacar las siguientes ideas:

1a . Respecto a la definición de mdivación, tras recoger las opiniones de Paris, Olson y Stevenson, 1983; Weiner, 1986; McClelland, 1987; Stipek, 1988, y otros, resumidas por González y Tourón (1992:285), podríamos entenderla como proceso que explica el inicio, dirección, intensidad y perseverancia de la conducta encaminada hacia el logro de una meta, modulado por las percepciones que los sujetos tienen de sí mismos y por las tareas a las que se tienen que enfrentar. Destacando como su elemento nuclear el autoconcepto.

$2^{\circledR}$. En cuanto a lasteorías sobre la motivación, aunque desde diferentes puntos de vista y tratando distintos aspectos, a veces, complementarios y enriquecedores, resaltar las aportaciones de Lepper, Greene y Nisbett (1973) sobre motivación intrínseca, la de Weiner (1974) sobre atribución causal, las de Eccles et al. (1983) sobre la expectativa-valor de la tarea, mejoradas por Pintrich y De Groot (1990), introduciendo como elementos determinantes los componentes expectativos, valorativos y afectivos -autoeficacia, valor intrínseco y control de la ansiedad-.

$3^{a}$. Sobre los instrumentos de evaluación de la motivación más utilizados, en principio, han sido las escalas Thurstone (1929) y Likert (1932), el escalograma de Gutman (1945), el diferencial semántico de Osgood (1951) y la escala de Barker (1969); si bien, más recientemente, se vienen utilizando el AMI de Doyle y Moen (1978), la escala de Harter (1980 y 1985), el SLRIS de Zimmerman y Martínez Pons (1986) o el MSLQ de Pintrich y De Groot (1990).

4a . Referente a los programas de intervención en motivación resaltar las aportaciones de McCleland (1974) y De Charms (1976) en motivación de logro, las de McAuley (1985) y Forsyth (1986) sobre intervención atribucional, las de Brophy y Kher (1986) sobre motivación para aprender, la de Stipek (1988) sobre motivación intrínseca, las de Ames y Ames (1991) sobre motivación de últimos resultados (tanto por parte del profesor como de los alumnos), las de Epstein (1989) y Ames 
(1990) sobre motivación en metas de logro y las de Alonso (1991) en motivación hacia metas de aprendizaje y metas de logro, que servirán de base para la elaboración del nuestro programa de intervención.

Todo lo cual nos lleva a decantarnos, por una parte, a la hora de evaluar la motivación (autoeficacia, valor intrínseco y control de la ansiedad), a través del cuestionario de Pintrich y De Groot (1990); y, por otra parte, respecto a la intervención con un programa propio de mejora, teniendo en cuenta las ideas aportadas por McCleland (1974), De Charms (1976), Weiner (1979); McAuley (1985), Forsyth (1986), Brophy y Kher (1986), Stipek (1988), Ames y Ames (1991), Epstein (1989), Alonso (1991), Ames (1992), y Hernández y García Hernández (1992).

\section{HIPÓTESIS}

Nuestra hipótesis general de trabajo ha quedado configurada de la siguiente forma: Las creencias motivacionales de los alumnos del contexto educativo pluricultural de Ceuta afectan a su rendimiento académico, difieren en función de su cultura origen y son mejorables con el programa de intervención adecuado.

A partir de ella, para mayor operatividad, se han podido formular las siguientes subhipótesis:

1a . Existen diferencias estadísticamente significativas en las creencias motivacionales «autoeficacia, valor intrínseco y ansiedad» de los alumnos de los cursos $4^{\circ}$ y $6^{\circ}$ de Educacón Primaria y $2^{\circ}$ de E.S.O., en función de su cultura -cristiana y musulmana-, aunque no de su sexo -hombre y mujer-, en el contexto educativo pluricultural de Ceuta.

$2^{0}$. Existen diferencias estadísticamente significativas en el rendimiento académico de los alumnos de los cursos 4ำ y $6^{\circ}$ de Educación Primaria y 20 de E.S.O., en función de su cultura -cristiana y musulmana-, aunque no de su sexo -hombre y mujer-, en el contexto educativo pluricultural de Ceuta.

$3^{0}$. Existen relaciones estadísticamente significativas entre las variables analizadas y que las creencias motivacionales predicen el rendimiento académico.

$4^{\circ}$. Una vez encontrados niveles bajos estadísticamente significativos en las creencias motivacionales que pudieran afectar al rendimiento académico, es posible elaborar y aplicar un programa de intervención para mejorar la situación y comprobar su eficacia.

\section{MUESTRA}

Los sujetos con los que se realizó esta investigación fueron 256 alumnos de los cursos $4^{\circ}$ y $6^{\circ}$ ce Educación Primaria y $2^{\circ}$ de E.S.O., de un Colegio Público de Ceuta extraído al azar, de entre aquellos que tienen el $50 \%$ de alumnos musulmanes y cristianos. La razón de la elección de un centro educativo que contara con igual distribución de alumnos, en función de sus culturas, se debió a que, en el resto, la mayoría de alumnos de una u otra cultura condiciona diferencias a su favor en autoconcepto -elemento nuclear de la motivación- y en rendimiento académico, especialmente en los casos más extremos (Ramírez Salguero, 1997).

\section{VARIABLES Y DISEÑO}


A) En el Pretest

\section{Dependiente}

\section{El rendimiento académico.}

\section{Independiente}

Las creencias motivacionales: autoeficacia, valor intrínseco y ansiedad.

B) En el Postest

\section{Dependiente}

La variable dependiente de esta investigación es son las creencias motivacionales: autoeficacia, valor instrínseco y ansiedad.

\section{Independiente}

La variable independiente utilizada en esta investigación es nuestro tratamiento experimental: Programa de intervención para el desarrollo de las creencias motivacionales -PIDCM- que expresamente hemos construido para la ocasión.

Todas estas variables, han sido tenidas en cuenta en función de su incidencia en la distribución de los siguientes factores:

$1^{\circ}$. Cursos: $4^{\circ}$ y $6^{\circ}$ deEducación Primaria y $2^{\circ}$ de E.S.O.

$2^{\circ}$. Grupos:experimental y control.

$3^{\circ}$. Sexos:hombre y mujer.

$4^{\circ}$. Culturas cristiana y musulmana.

El diseño empleado ha sido el experimental con pretest-postest, tipo 6 de Campbell y Stanley (1966).

\section{PROCEDIMIENTO E INSTRUMENTOS}

El procedimiento consistió en analizar en la muestra elegida, en primer lugar, los niveles y relaciones existentes entre las variables, para, en vista de su idoneidad, pasar a elaborar y aplicar un tratamiento de mejora de las creencias motivacionales. Para ello, en segundo lugar, se dividió aleatoriamente la muestra en dos grupos, -experimental y control- por cursos, para llevar a cabo un contraste postest, tras seis meses de tratamiento (23 sesiones o semanas). Para ello, hubo que instruir convenientemente a 12 monitores, encargados de su aplicación.

La evaluación de la variable creencias motivacionales: autoeficacia, valor intrínseco y ansiedad, se ha hecho a través de la aplicación del cuestionario MSLQ de Pintrich y De Groot (1990). Variable que, operativamente, podría quedar definida como conjunto de autovaloraciones integrado por los componentes de autoeficacia, valor intrínseco y ansiedad, que activan, dirigen y mantienen la conducta de los alumnos. Entendiendo, a su vez, por autoeficacia, la apreciación del alumno acerca de sus posibilidades al enfrentarse con el aprendizaje; por valor intrínseco, la apreciación del alumno respecto hacia dónde dirige su esfuerzo, sus gustos o preferencias y la escala de valores por los que se rige; y, por ansiedad, la apreciación del 
alumno referente al estado emocional que desarrolla al enfrentarse a los exámenes.

Por otra parte, la variable rendimiento académico se ha evaluado utilizando las calificaciones de los alumnos, otorgadas por los profesores.

En cuanto al Programa de Intervención para el Desarrollo de las Creencias Motivacionales -PIDCM-, a continuación se exponen sus rasgos descriptivos más significativos:

\section{Introducción}

La atención al ámbito afectivo, individual y social, es uno de los objetivos más enfatizados por los especialistas de la educación y las leyes educativas actuales, hasta el extremo que le reconocen el mismo valor, o incluso por encima, que a los objetivos estrictamente académicos; no obstante, parece ser que su tratamiento específico en el desarrollo del currículo no queda concretado de una forma clara, siendo abordados ocasionalmente en la mayor parte de las casos.

Así pues, este programa tiene como objetivo primordial desarrollar la motivación de los niños y jóvenes, potenciando su ajuste personal y socialización a través del concepto positivo de sí mismos (autoeficacia y valor intrínseco) y del control de su ansiedad, fomentando la tolerancia, la comprensión, la comunicación y la cooperación con los demás, para la superación de los problemas que la vida plantea a las personas, tanto en la escuela, como en la familia, el grupo de amigos y la sociedad en general.

\section{Descripción general}

Los principales rasgos descriptivos de este programa son los siguientes:

* Nombre: "Programa de Intervención para el Desarrollo de las Creencias Motivacionales - PIDCM".

* Destinatarios: Alumnos de Segundo y Tercer Ciclo de Educación Primaria y de ESO.

* Fundamentación: Se considera que la falta de motivación suficiente tiene su raíz en la manera inadecuada de canalizar las emociones, creando desajustes afectivos. La base teórica para la elaboración de este programa de intervención se encuentra en los trabajos de McCleland (1974) y De Charms (1976), en motivación de logro; los de McAuley (1985) y Forsyth (1986), sobre intervención atribucional; los de Brophy y Kher (1986), en motivación para aprender; el de Stipek (1988) sobre motivación intrínseca; los de Ames y Ames (1991), en motivación de últimos resultados; los de Epstein (1989), Alonso (1991) y Ames (1992), sobre motivación en metas de aprendizaje y metas de logro; los de Pintrich y De Groot (1990), en creencias motivacionales; y los de Hernández y García Hernández (1992), sobre educación y liberación emotiva.

* Procedimientos:

Materiales: Hojas guía y de respuesta.

- Tipo de aplicación: Individual y colectiva (pequeños grupos).

- Tiempo de aplicación: 23 semanas, a sesión por semana.

- Actividades: Lectura silenciosa y en voz alta, respuestas orales y escritas, discusión en grupo, psicodrama y ejemplificación, y realización de compromisos.

* Estructuración: 
1. Unidades: Este programa está constituido por cinco unidades:

I. En busca del tesoro.

II. Cómo desarrollar mi autoeficacia.

III. Cómo desarrollar mi valor intrínseco.

IV. Cómo controlar mi ansiedad.

V. Revisión de lo trabajado y planes de futuro.

2. Secciones: Cada unidad está constituida por distintas secciones y cada sección suele recoger los siguientes aspectos: (1) Ideas principales. (2) Narraciones. (3) Elaboración por escrito. (4) Discusiones.

3. Sesiones: En cada sesión pueden desarrollarse una o dos secciones, atendiendo al tiempo disponible y al ritmo del grupo.

4. Unidades y secciones:

I. En busca del tesoro.

1. Cada día puede ser una aventura.

2. En la vida hay un tesoro muy importante que descubrir.

3. El mejor tesoro está muy cerca de ti.

4. El que sabe vivir es feliz.

5. El viejo libro puede enseñarte a conocerte mejor y aprender a vivir.

II. Cómo desarrollar mi autoeficacia.

1. El concepto que tengo de mí es fundamental para ser feliz.

2. Las personas terminan siendo lo que creen que son.

3. Las personas terminan siendo lo que los demás creen de ellas.

4. Las personas que no se valoran no son felices.

5. Mis pensamientos pueden cambiar mi forma de ser y de comportarme.

6. Las personas alegres y felices lo son porque saben disfrutar de todo lo que tienen.

7. Muchas veces ocurren cosas contrarias a nuestros deseos.

8. Las personas felices aceptan lo que no tienen remedio, pero luchan por las cosas que tienen solución.

9. Siempre hay fallos. Pero, ¿qué hay que hacer para fallar cada menos?.

10. Para tener éxito hay que descubrir la importancia e interés de lo que uno hace.

11. Para tener éxito hay que pensar antes de actuar.

12. Para tener éxito hay que tener claros los objetivos, buscar los medios e imaginar las consecuencias 
13. Para tener éxito hay que estar atento.

III. Cómo desarrollar mi valor intrínseco.

1. El colegio es como mi segunda casa.

2. Comprender es importante para aprender.

3. Lo que aprendo en el colegio, si yo quiero, puede servir para mi vida.

4. El saber es disfrute y poder.

5. El esfuerzo diario en clase garantiza más ventajas y satisfacciones para el futuro.

6.Lo que ahora nos cuesta, puede convertirse en un placer.

7. Las cosas que parecen muy difíciles se pueden alcanzar si nos lo proponemos.

8. Hay que darse ánimos para conseguir lo que deseamos.

9. Las cosas se consiguen poco a poco, no al momento.

10. Cuando conseguimos lo que nos proponemos debemos sentirnos satisfechos de nosotros mismos.

IV. Cómo controlar mi ansiedad.

1. El miedo inventa cosas terribles.

2. El miedo lo provoca nuestra imaginación, nuestra fantasía.

3. El miedo lo provoca también mi propia inseguridad.

4. Las cosas se ven peor cuando sentimos miedo.

5. Tenemos que animarnos cuando sintamos miedo.

6. En la vida ocurren cosas agradables.

7. Si somos tristes y aburridos es porque sufrimos pensando en lo que no tenemos, en lo que no podemos conseguir.

8. Las personas que se obsesionan con cosas imposibles nunca son felices.

9. En nuestra vida podemos tener fracasos y fallos.

10. Los fallos pueden ser con las personas o con lo que hacemos.

11. Cuando la gente fracasa puede tener buenas reacciones o reacciones inútiles.

V. Revisión de lo trabajado y planes de futuro.

1. Individual.

2. Grupal.

\section{TÉCNICAS DE ANÁLISIS}

El tratamiento de los datos se ha llevado a cabo, mediante el Statistickal Package for Social Sciences (SPSS-11), en tres niveles: 
1․ . Nivel descriptivo.

Este nivel introductorio de análisis, el más simple, aunque no el menos importante, facilita el conocimiento de las características del estudio, una vez depurados los datos, analizando las propiedades más relevantes que servirán como punto de partida a los análisis posteriores. Con tal fin, se han utilizado las medidas de tendencia central, representativas del conjunto de la distribución y las de variabilidad, para saber cómo se agrupan los datos.

\section{$2^{0}$. Nivel inferencial.}

La estadística inferencial delimita qué diferencias de las halladas se deben al azar y cuáles no; así pues, el estadístico utilizado en este nivel ha sido el Análisis de varianza , ya que las pruebas de homocedasticidad así lo indicaban. Los análisis posteriores se llevaron a cabo con el procedimiento de cálculo denominado $L S D$, o prueba de las diferencias mínimas significativas, que es la menos conservadora de las pruebas disponibles a posteriori en el análisis de varianza. El nivel de confianza fijado ha sido del 95\%, para las afirmaciones que se realizan, y, en aquellos casos que este nivel se rebasa, se indica oportunamente.

\section{$3^{\circ}$. Nivel multivariable}

El estadístico usado en este nivel ha sido el Análisis multifactorial de covarianza, que combina las técnicas de análisis de varianza y regresión, tratándose de un modelo lineal general multivariado en el que es posible contrastar hipótesis nulas respecto a los efectos de los factores sobre las medias de varias agrupaciones de una distribución conjunta de variables dependientes. Asimismo, puede investigar las interacciones entre los factores y sus efectos individuales. Por otra parte, se pueden incluir los efectos de las covariables y las interacciones de éstas con los factores. Para el análisis de regresión, las variables independientes (predictoras) se especifican como covariables. La decisión de elegir este estadístico se debe a que se ajusta al tipo de diseño planificado en nuestro estudio con distintas variables concomitantes.

\section{RESULTADOS}

\section{ANÁLISIS PRETEST}

El análisis pretest se ha llevado a cabo para comprobar el estado inicial de las variables sometidas a estudio, hallándose los siguientes resultados:

\subsection{Por cursos}

El análisis descriptivo, inferencial y multivariable de las creencias motivacionales en el pretest, pone de manifiesto lo siguiente:

a) En la variable autoeficacia, los cuartos cursos obtienen la mayor media ( $=49,39$ ), por encima de los sextos $(=49,26)$ y los octavos $(=47,45)$, hallándose diferencias estadísticamente signficativas entre ellos en general ( $F=4,747$ - sig. ,009), y, a través de comparaciones múltiples, tan sólo entre los cuartos y los octavos (sig. ,006), y los sextos y octavos (sig. ,012), en favor de los primeros respectivamente. Poniendo de relieve qué cursos se consideran más autoeficaces.

b) En la variable valor intrínseco, los sextos cursos obtienen la mayor media ( $=50,79$ ) por encima de los octavos ( $=46,94)$ y los cuartos $(=44,39)$, hallándose diferencias estadísticamente signficativas 
entre ellos en general $(F=18,535$ - sig. ,000), y, a través de comparaciones múltiples, entre los sextos y los cuartos (sig. ,000), los sextos y octavos (sig. ,000) y los octavos y cuartos (sig. ,015), en favor de los primeros respectivamente. Poniendo de relieve qué cursos se consideran con mayor valor intrínseco.

c) En la variable ansiedad, los sextos cursos obtienen la mayor media ( $=12,93)$, por encima de los octavos ( $=12,00)$ y los cuartos $(=10,72$ ), hallándose diferencias estadísticamente signficativas entre ellos en general $(F=25,288$ - sig. ,000), y, a través de comparaciones múltiples, entre los sextos y los cuartos (sig. ,000), los sextos y octavos (sig. ,000) y los octavos y cuartos (sig. ,000), en favor de los primeros respectivamente. Poniendo de relieve qué cursos se consideran los más ansiosos.

$\mathrm{Y}$, el análisis descriptivo, inferencial y multivariable del rendimiento académico en el pretest, revela que los cuartos cursos obtienen la mayor media $(=8,53)$, por encima de los octavos $(=7,83)$ y los sextos ( = 6,97), hallándose diferencias significativas entre ellos en general $(F=3,153$ - sig. ,044), y, a través de comparaciones múltiples, tan sólo entre los cuartos y los octavos (sig. ,014), en favor de los primeros. Poniendo de relieve que las mejores puntuaciones obtenidas en rendimiento académico corresponden a los alumnos de los cuartos cursos.

\subsection{Por sexos}

El análisis descriptivo, inferencial y multivariable de cada una de las variables tratadas en el pretest, creencias motivacionales y rendimiento académico, por sexos, en general, pone de manifiesto que no existen diferencias estadísticamente significativas en la mayoría de ellas, salvo en el caso concreto de la autoeficacia ( $F=4,127$ - sig.,043), a favor de las chicas «mujer ( $=49,28)$, varón ( $=48,10)$ ».

\subsection{Por culturas}

El análisis descriptivo, inferencial y multivariable de las creencias motivacionales por culturas en el pretest, pone de manifiesto que existen diferencias estadísticamente significativas en el siguiente sentido:

a) En la variable autoeficacia, aunque la media obtenida por los cristianos ( $=48,94$ ) es superior a la de los musulmanes ( $=48,42)$, no se han encontrado diferencias sigificativas entre ellas $(F=, 791$ - sig. ,375). Poniendo de relieve que ambos se consideran igualmente autoeficaces.

b) En la subvariable valor intrínseco, aunque la media obtenida por los cristianos ( $=47,47$ ) es \$perior a la de los musulmanes $(=47,11)$, no se han encontrado diferencias significativas entre ellas $(\mathrm{F}=$ ,152 - sig. ,697). Poniendo de relieve que ambos consideran tener el mismo valor intrínseco.

c) En la subvariable ansiedad, los musulmanes obtienen la mayor media ( $=12,26$ ), por encima de los cristianos ( $=11,50$ ), hallándose diferencias signficativas entre ellos $(F=7,641$ - sig. ,006). Poniendo de relieve que los musulmanes se muestran más ansiosos.

Y el análisis descriptivo, inferencial y multivariable del rendimiento académico por culturas en el pretest, muestra cómo los cristianos obtienen una media $(=9,02)$ superior a la de los musulmanes $(=$ $6,50)$, hallándose diferencias significativas entre ellos $(F=25,210$ - sig. ,000). Poniendo de relieve que los cristianos muestran mejor rendimiento académico.

\subsection{Predicción del rendimiento académico}

Respecto a la predicción de las diferentes variables tratadas sobre el rendimiento académico en el pretest, se ha empleado el método de regresión múltiple paso a paso "stepwise", considerando como varia- 
ble criterio el rendimiento académico y como variables predictoras todas las demás. Fijándonos en el último paso y, dentro de él, en el orden de entrada de las variables en la ecuación de regresión, en el valor de cada variable, en su significación, y, a nivel más general, en el índice de regresión $(R)$ y en el coeficiente de determinación múltiple $\left(\mathrm{R}^{2}\right)$, que indica el porcentaje de varianza explicada por el conjunto de las variables predictoras incluidas en la ecuación, los resultados obtenidos han sido los siguientes (Tablas 1 y 2).

Tabla 1

Regresión múltiple paso a paso "stepwise", considerando como variable criterio el rendimiento académico y como variables predictoras el curso y la cultura.

\begin{tabular}{|c|c|c|c|c|}
\hline $\mathbf{R}=\mathbf{0 , 3 3 9}$ & $R^{2}=$ & & $F^{(2-256)}=1$ & \\
\hline Variables & B & $\beta$ & $t$ & p \\
\hline Constante general & 13,959 & & 11,701 & 0,000 \\
\hline Curso & $-0,40$ & $-0,157$ & $-2,653$ & 0,008 \\
\hline Cultura & $-2,54$ & $-0,302$ & $-5,107$ & 0,000 \\
\hline
\end{tabular}

Tabla 2

Regresión múltiple paso a paso "stepwise", considerando como variable criterio el rendimiento académico y como variables predictoras las creencias motivacionales en el pretest.

\begin{tabular}{|c|c|c|c|c|}
\hline $\mathbf{R}=0,424$ & \multicolumn{1}{c|}{$\mathbf{R}^{2}=0,180$} \\
$p=0,000$ & $F^{(2-256)}=27,760$ \\
\hline Variables & B & $\beta$ & $t$ & $p$ \\
\hline Constante general & 6,000 & & 2,037 & 0,003 \\
\hline Ansiedad & $-0,649$ & $-0,343$ & $-6,000$ & 0,000 \\
\hline Autoeficacia & 0,196 & 0,217 & 3,789 & 0,000 \\
\hline
\end{tabular}

Tomando los resultados recogidos en la tabla 1 se observa que en la comunidad escolar ceutí, el Rendimiento Académico Global de los alumnos está determinado por las variables siguientes: "curso" y "cultura"; quedando excluido el sexo. El conjunto de las variables "curso" y "cultura" dan cuenta del 11,57\% de la varianza en el rendimiento académico $\left(R^{2}=0,1157\right)$, si bien el mayor poder determinante lo ejerce el factor "cultura", que explica el $30,20 \%$ de la varianza en el rendimiento académico global.

La función descrita sería la siguiente:

\section{Rendimiento Académico Global $=13,959-0,400$ curso $-2,540$ cultura}

Tomando los resultados recogidos en la tabla 2 se observa que en la comunidad escolar ceutí, el Rendimiento Académico Global de los alumnos está determinado por las variables siguientes: "ansiedad" y "autoeficacia", quedando excluida la variable "valor intrínseco". El conjunto de las variables "ansiedad" y "autoeficacia" dan cuenta del $18,00 \%$ de la varianza en el rendimiento académico $\left(R^{2}=0,180\right)$, si bien el mayor poder determinante lo ejerce la variable "ansiedad", que explica el $34,30 \%$ de la varianza en el rendimiento académico global, pero en sentido negativo; es decir, contrario al rendimiento, como es lógico.

La función descrita sería la siguiente: 


\section{Rendimiento Académico Global $=6,000-0,649$ ansiedad + 0,196 autoeficacia}

\section{ANÁLISIS PRETEST-POSTEST}

El análisis pretest-postest se ha realizado para comprobar los efectos del tratamiento experimental aplicado, hallándose los siguientes resultados:

\subsection{Creencias motivacionales}

\subsubsection{Autoeficacia}

El análisis descriptivo, inferencial y multivariable pretest-postest de esta variable, pone de manifiesto lo siguiente:

a) Se aprecian diferencias estadísticamente significativas entre los grupos ( $F=38,771$ - sig. ,000), obteniendo los mejores resultados los grupos experimentales ( $=49,93)$, a los que se les aplicó el tra由miento experimental en motivación, seguidos por los grupos de control ( $=48,62)$.

b) Se observan diferencias estadísticamente significativas entre los cursos por culturas ( $F=3,665$ sig. ,027), obteniendo los mejores resultados los cristianos de los cuartos cursos ( = 51,73), seguidos por los cristianos de los sextos cursos ( $=50,76)$, los musulmanes de los sextos cursos $(=50,27)$, los musulmanes de los cuartos cursos ( $=50,00$ ), los musulmanes de losoctavos cursos ( $=49,68$ ) y los cristianos de los octavos cursos ( $=49,47)$.

\subsubsection{Valor intrínseco}

El análisis descriptivo, inferencial y multivariable pretest-postest de esta variable, pone de manifiesto lo siguiente:

a) Se aprecian diferencias estadísticamente significativas entre los cursos $(F=12,081$ - sig. ,000), obteniendo los mejores resultados los sextos cursos $(=51,82)$, seguidos de los cuartos cursos $(=50,28)$, y los octavos cursos ( $=49,33)$.

b) Se aprecian diferencias estadísticamente significativas entre los grupos ( $F=31,739$ - sig. ,000), obteniendo los mejores resultados los grupos experimentales ( = 49,81), a los que se aplicaron el tra由miento experimental en motivación, seguidos por los grupos de control ( $=49,33$ )

c) Se aprecian diferencias estadísticamente significativas entre los cursos por grupos y sexos ( $F=$ 16,791 - sig. ,016), obteniendo los mejores resultados las chicas de los sextos cursos, grupos experimentales ( $=53,29$ ); seguidos porlos chicos de los sextos cursos, grupos experimentales $(=51,64)$; etc.

d) Se aprecian diferencias estadísticamente significativas entre los cursos por culturas $(F=7,518$ sig. , 016), obteniendo los mejores resultados los cristianos de los sextos cursos ( = 5209), seguidos por los musulmanes de los sextos cursos ( $=51,49)$, los cristianos de los cuartos cursos( $=51,00)$, los mus $U_{r}$ manes de los octavos cursos ( $=49,54$ ), los musulmmes de los cuartos cursos ( $=49,53$ ), y los cristianos de los octavos cursos $(=49,13)$.

e) Se aprecian diferencias estadísticamente significativas entre los cursos por grupos y culturas ( $F=$ 3,880 - sig. ,005), obteniendo los mejores resultados los cristianos de los sextos cursos de los grupos experimentales ( $=52,56)$;los musulmanes de los sextos cursos de los grupos experimentales $(=52,33)$; etc. 


\subsubsection{Ansiedad}

El análisis descriptivo, inferencial y multivariable pretest-postest de esta variable, pone de manifiesto lo siguiente:

a) Se aprecian diferencias estadísticamente significativas entre los grupos ( $F=37,894$ - sig. ,000), obteniendo los resultados más altos los grupos de control ( $=12,00)$, seguidos de los grupos experim€tales $(=8,94)$, a los que se aplicó sólo el tratamiento experimental ๓ motivación.

b) Se aprecian diferencias estadísticamente significativas entre los cursos por grupos $(F=3,672$ sig. ,006), obteniendo los resultados más altos los sextos cursos, grupos de control ( =12,52); seguidos de los octavos cursos, grupos de control ( $=12,00)$; los cuartos, grupos de control ( $=11,50)$; los sextos cuf sos, grupos experimentales; etc.

c) Se aprecian diferencias significativas entre los cursos por grupos y sexos ( $F=3,358$ - sig. ,011), obteniendo los resultados más altos los chicos de los sextos cursos, grupos de control ( =12,85); $\mathbf{8}$ guidos por las chicas de los sextos cursos, grupos de control ( $=12,21)$; las chicas de los octavos cursos, grupos de control ( $=12,13$ ); los chicos de los octavos cursos, grupos de control ( $=11,87$ ); las chicas de los cuartos cursos, grupos de control $(=11,86)$; los chicos de los cuatos cursos, grupos de control $(=11,14)$; etc.

d) Se aprecian diferencias significativas entre los grupos por culturas ( $F=4,165$ - sig. ,017), obteniendo los resultados más altos los musulmanes de los grupos de control ( =12,19); seguidos por los cristianos de los grupos de control ( = 11,81); los musulmanes de los grøos experimentales $(=9,00)$; los cristianos de los grupos experimentales.

\subsection{Rendimiento académico}

El análisis descriptivo, inferencial y multivariable pretest-postest de esta variable, pone de manifiesto lo siguiente:

a) Se aprecian diferencias estadísticamente significativas entre los grupos $(F=37,179$ - sig. ,000), obteniendo los mejores resultados los grupos experimentales $(=10,63)$ a los que se aplicó el tratamiento experimental en motivación, seguidos por los grupos de control $(=8,85)$.

b) Se aprecian diferencias estadísticamente significativas entre las culturas ( $F=4,899$ - sig. ,028), obteniendo los mejores resultados los cristianos $(=11,36)$, segudos por los musulmanes $(=9,04)$.

c) Se aprecian diferencias estadísticamente significativas entre los cursos por grupos $(F=3,447$ sig. ,009), obteniendo los mejores resultados los cuartos cursos grupos experimentales $(=11,03)$;los sextos cursos, grupos experimentales $(=10,54)$; los octavos cursos, grupos experimentales $(=10,26)$; los cuartos cursos, grupos de control $(=10,11)$; los octavos cursos, grupos de control $(=8,27)$ y los sextos cursos, grupos de control ( $=8,19)$.

\subsection{Predicción del rendimiento académico}

Respecto a la predicción de las diferentes variables tratadas sobre el rendimiento académico en el postest, se ha empleado el método de regresión múltiple paso a paso "stepwise", considerando como variable criterio el rendimiento académico y como variables predictoras todas las demás. Fijándonos en el último paso y, dentro de él, en el orden de entrada de las variables en la ecuación de regresión, en el valor de ca- 
da variable, en su significación, y, a nivel más general, en el índice de regresión $(R)$ y en el coeficiente de determinación múltiple $\left(\mathrm{R}^{2}\right)$, que indica el porcentaje de varianza explicada por el conjunto de las variables predictoras incluidas en la ecuación, los resultados obtenidos han sido los siguientes (Tabla 3).

\section{Tabla 3}

Regresión múltiple paso a paso "stepwise", considerando como variable criterio el rendimiento académico y como variables predictoras las creencias motivacionales en el postest.

\begin{tabular}{|c|c|c|c|c|}
\hline $\mathbf{R}=0,478$ & \multicolumn{1}{c|}{$\mathbf{R}^{2}=0,228$} \\
$p=0,000$ & $F^{(2-256)}=37,386$ \\
\hline Variables & B & $\beta$ & $t$ & $p$ \\
\hline Constante general & $-6,064$ & & $-1,597$ & 0,012 \\
\hline Ansiedad & $-0,294$ & $-0,287$ & $-4,980$ & 0,000 \\
\hline Autoeficacia & 0,382 & 0,309 & 5,360 & 0,000 \\
\hline
\end{tabular}

Tomando los resultados recogidos en la tabla 3 se observa que en la comunidad escolar ceutí, el Rendimiento Académico Global de los alumnos está determinado por las variables siguientes: "ansiedad" y "autoeficacia", quedando excluida la variable "valor intrínseco". El conjunto de las variables "ansiedad" y "autoeficacia" dan cuenta del $22,80 \%$ de la varianza en el rendimiento académico $\left(R^{2}=0,228\right)$, si bien el mayor poder determinante lo ejerce la variable "autoeficacia", que explica el $30,90 \%$ de la varianza en el rendimiento académico global.

La función descrita sería la siguiente:

\section{Rendimiento Académico Global = -6,064 - 0,294 ansiedad + 0,382 autoeficacia}

Finalmente, si observamos las diferencias entre los índices de las tablas 2 y 3, podremos comprobar las diferencias pretest - postest en las funciones de regresión, que nos muestran como tras la aplicación del tratamiento experimental en desarrollo de las creencias motivacionales, se reduce el poder predictor de la ansiedad en el rendimiento académico y aumenta el de la autoeficacia; lo cual, de alguna forma, certifica la bondad del tratamiento.

\section{CONCLUSIONES}

Así pues, en función de los resultados obtenidos, se han podido deducir las siguientes conclusiones:

I. Respecto a la hipótesis general: Las creencias motivacionales de los alumnos del contexto educativo pluricultural de Ceuta afectan a su rendimiento académico, difieren en función de su cultura origen y son mejorables con el programa de intervención adecuado, decir que se confirma casi totalmente, salvo en el caso de la variable valor intrínseco, de las creencias motivacionales, siendo siempre los musulmanes los más afectados.

\section{Respecto a las subhipótesis:}

$1^{\mathrm{a}}$. Existen diferencias estadísticamente significativas en las creencias motivacionales «autoeficacia, valor intrínseco y ansiedad» de los alumnos de los cursos $4^{\circ}$ y $6^{\circ}$ de Educacón Primaria y $2^{2}$ de E.S.O, en función de su cultura -cristiana y musulmana-, aunque no de su sexo-hombre y mu- 
jer-, en el contexto educativo pluricultural de Ceuta.

A) En el Pretest

10. Por cursos.

En la subvariable autoeficacia, los cuartos de Educación Primaria obtienen los mejores resultados.

En la subvariable valor intrínseco, los sextos cursos de Educación Primaria obtienen los mejores resultados.

En la subvariable ansiedad, los sextos cursos de Educación Primaria resultan ser los más ansiosos.

\section{$2^{2}$. Por sexos.}

Se pone de manifiesto, en general, que no existen diferencias significativas en la mayoría de las variables tratadas, salvo en el caso concreto de la autoeficacia, en la que obtienen mejores resultados las chicas.

\section{$3^{\circ}$. Por culturas.}

En la subvariable autoeficacia, no se han encontrado diferencias estadísticamente significativas.

En la subvariable valor intrínseco, no se han encontrado diferencias estadísticamente significativas.

En la subvariable ansiedad, existen diferencias estadísticamente significativas, mostrándose más ansiosos los musulmanes.

\section{B) En el Postest}

\section{Por cursos.}

En la subvariable autoeficacia, los cuartos de Educación Primaria del grupo experimental obtienen los mejores resultados.

En la subvariable valor intrínseco, los sextos cursos de Educación Primaria del grupo experimental obtienen los mejores resultados.

En la subvariable ansiedad, los sextos cursos de Educación Primaria del grupo de control resultan ser los más ansiosos.

\section{2o . Por sexos.}

Se pone de manifiesto, en general, que no se han hallado diferencias estadísticamente significativas.

\section{$3^{\circ}$. Por culturas.}

En la subvariable autoeficacia, se han encontrado diferencias estadísticamente significativas a favor de los cristianos de los grupos experimentales.

En la subvariable valor intrínseco, se han encontrado diferencias estadísticamente significativas a favor de los cristianos de los grupos experimentales.

En la subvariable ansiedad, existen diferencias estadísticamente significativas, mostrándose más 
ansiosos los musulmanes de los grupos de control.

\section{$4^{\circ}$. Por grupos.}

En la subvariable autoeficacia, se han encontrado diferencias estadísticamente significativas a favor de los grupos experimentales; es decir, aquellos a los que se les aplicó el tratamiento.

En la subvariable valor intrínseco, se han encontrado diferencias estadísticamente significativas a favor de los grupos experimentales; es decir, aquellos a los que se les aplicó el tratamiento.

En la subvariable ansiedad, existen diferencias estadísticamente significativas, mostrándose más ansiosos los grupos de control.

$2^{\circ}$. Existen diferencias estadísticamente significativas en el rendimiento académico de los alumnos de los cursos $4^{\circ}$ y $6^{\circ}$ de Educaión Primaria y $2^{\circ}$ de E.S.O, en función de su cultura -cristiana y musulmana-, aunque no de su sexo -hombre y mujer-, en el contexto educativo pluricultural de Ceuta.

\section{A) En el Prestest}

No se han encontrado diferencias estadísticamente significativas en función del sexo, pero sí en función de la cultura, a favor de los alumnos cristianos.

\section{B) En el Postest}

No se han encontrado diferencias estadísticamente significativas en función del sexo, pero sí en función de la cultura, a favor de los alumnos cristianos de los grupos experimentales, encabezados por los de cuarto curso de Educación Primaria.

$3^{\circ}$. Existen relaciones estadísticamente significativas entre las variables analizadas y que las creencias motivacionales predicen el rendimiento académico.

\section{A) En el Prestest}

Al rendimiento académico lo predicen los factores cultura y curso, en ese orden, y, asimismo, las variables ansiedad y autoeficacia.

\section{B) En el Postest}

Aunque, al rendimiento académico lo siguen prediciendo las variables ansiedad y autoeficacia, se observa que ha aumentado significativamente la autoeficacia y se ha reducido la ansiedad, en general, y, en particular, en el caso de los grupos experimentales.

$4^{\circ}$. Una vez encontrados niveles bajos estadísticamente significativos en las creencias motivacionales que pudieran afectar al rendimiento académico, es posible elaborar y aplicar un programa de intervención para mejorar la situación y comprobar su eficacia.

Efectivamente, hallados niveles bajos, estadísticamente significativos, en las creencias motivacionales que afectaban al rendimiento académico, se diseño y desarrollo un programa de intervención -PIDCMque ha conseguido los siguientes logros:

1ํ. Los cursos más beneficiados han sido los cuartos y sextos de Educación Primaria.

$2^{\circ}$. Los grupos experimentales han mejorado significativamente sus niveles de autoeficacia y valor 
intrínseco, y han reducido su ansiedad, en lo referido a las creencias motivacionales.

$3^{o}$. Entre los sexos, la única diferencia significativa que existía, en su autoeficacia -a favor de las chicas- ha quedado neutralizada.

$4^{\circ}$. Las diferencias entre culturas se han reducido significativamente en el caso de las creencias motivacionales, especialmente, en la elevada ansiedad que mostraban los musulmanes. Por otra parte, el rendimiento académico se ha mejorado, en general, reduciendo las diferencias existentes entre las medias de los cristianos y musulmanes, aunque no de manera significativa.

Todas estas conclusiones hacen pensar en la idoneidad de la aplicación en las aulas de programas de intervención específicos en las variables tratadas para reducir los efectos negativos.

\section{BIBLIOGRAFÍA}

Abascal, J., Modelos de motivación. En Trianes, M.J. et al., Psicología de la Educación para profesores. Madrid, EUDEMA, 225-242, 1995.

Abascal, J., Motivación intrínseca y autodesarrollo. En Trianes, M.J. et al., Psicología de la Educación para profesores. Madrid, EUDEMA, 243-260, 1995.

Alonso, J., Atribución de la causalidad y motivación de logro: Estudio evolutivo de la utilización de información en la realización de juicios de atribuciones. Estudios de Psicología, 16, 1983, 13-27.

Alonso, J., Atribuciones y conducta: una línea de investigación. Revista de Ciencias de la Educación, 126, 1986, 137-140.

Alonso, J., Estudio sobre la validez de constructo de los cuestionarios MAPE, EAT y ECO. Estudios de Psicología, 30, 1987, 45-69.

Alonso, J., ¿Enseñar a pensar?: Perspectivas para la Educación Compensatoria. Madrid, M.E.C., 1987.

Alonso, J., Motivación y aprendizaje en el aula. Madrid, Santillana, 1991.

Alonso, J., Motivar en la adolescencia: teoría, evaluación e intervención. Madrid, Servicio de Publicaciones de la Universidad Autónoma, 1992.

Alonso, J., ¿Qué es mejor para motivar a mis alumnos?. Análisis de lo que los profesores saben, creen y hacen al respecto. Madrid, Universidad Autónoma, 1993.

Alonso, J., Contexto, motivación y aprendizaje. En Alonso, J. y Caturla, E., La motivación en el aula. Madrid, PPC, 1996.

Alonso, J., Motivar en la adolescencia: teoría, evaluación e intervención. Madrid, Universidad Autónoma, 1992.

Alonso, J. y Montero, I., Motivación y aprendizaje escolar. En C. Coll, J. Palacios y A. Marchesi (Comps.), Desarrollo psicológico y educación. II: Psicología de la educación. Madrid: Alianza, 1990, 183-198.

Ames, C., Motivation. what teachers need to know. Teachers College Records, 91, 1990, 409-421.

Ames, R. \& Ames, C., Motivation and effective teaching. En L. Idol y B.F. Jones (Eds.): Educational values and cognitive instruction: implications for learning. Hillsdale, Erlbaum, 1991, 247-271. 
Atkinson, J.W., Theory of achievement motivation. New York, John Wiley, 1978.

Ball, S., La motivación educativa. Madrid, Narcea, 1988.

Beltrán, J.A., Psicología educacional. Madrid, UNED, 1984.

Beltrán, J.A. (Coord.), Psicología de la educación. Madrid, Eudema, 1987.

Brophy, J.E. \& Kher, N., Teacher socialization as a mechanism for developing student motivation to learn. En R.S. Feldman (Ed.): The social psychology of education. Cambridge, Cambridge University Press, 1986, 257-288.

Campbell, D. \& Stanley, J., Experimental and quasi-experimental desings for research. Chicago, Rand McNally \& Co., 1966 (Trad. 1973, Buenos Aires: Amorrortu).

De Charms, R., Enhancing motivation change in the classroom. New York, John Wiley, 1976.

Eccles, J., Expectancies, values, and academic behaviors. En J.T. Spence (Ed.), Achievement and achievement motives. San Francisco, Freeman, 1983, 75-146.

Epstein, J.L., Family structures and student motivation: a developmental perspective. En C. Ames y R. Ames (Eds.): Research on motivation in education: goals and cognitions. San Diego: Academic Press, Vol. 3, 1989, 259-285.

Forsyth, D.R., An attributional analysis of students' reactions to success and failure. En R.S. Feldman (Ed.): The social psychology of education. Cambridge, Cambridge University Press, 1986, 17-38.

González, M.C. y Tourón, J., Autoconcepto y rendimiento escolar. Pamplona, EUNSA, 1992, 388.

Lepper, M.R.; Greene, D. \& Nisbet, R.E., Undermining children's intrinsic interest with extrinsic rewards: a test of the "overjustification hypothesis". Journal of Personality and Social Psychology, 28, 1996, 129-137.

McClelland, D.C., Human motivation. Cambridge, Cambridge University Press, 1987 [Trad. (1989): Estudio de la motivación humana. Madrid, Narcea].

Paris, S.G.; Olson, G.M. \& Stevenson, H.M., Learning and motivation in the classroom. Hillsdale: Erlbaum, 1983.

Pintrich, P.R. \& De Groot, E.V., Motivational and self-regulated learning components of classroom academic performance. Journal of Educational Psychology, 82, 1990, 33-40.

Stipek, D.J., Motivation to learn: from theory to practice. New Yersey, Prentice Hall, 1988.

Toates, F., Motivational systems. Cambridge University Press, Cambridge, 1986 [Trad. (1989): Sistemas motivacionales. Madrid, Debate].

Weiner, B., An attributional theory of motivation and emotion. New York, Springer-Verlag, 1986.

Weiner, B., History of motivational research in education. Journal of Educational Psychology, 82, 1990, 616-622.

Zimmerman. B.J. \& Martínez-Pons, M., Development for a structure interview for assessing student use of self-regulated learning-strategy. American Educational Research Journal, 23, 1986, 614-628. 


\title{
Contactar
}

Revista lberoamericana de Educación

\author{
Principal OEI
}

